\title{
Biografía e historia de los intelectuales. Balance y reflexiones sobre la vida cultural argentina entre 1860 y $1910^{*}$
}

\section{Paula Bruno*}

\section{Resumen}

Este artículo presenta algunas reflexiones sobre una experiencia de investigación en la que se abordó la vida cultural argentina de la segunda mitad del siglo XIX y comienzos del siglo XX por medio de biografías comparadas. Se analizan las posibilidades de revisar las sincronías entre momentos históricos, períodos consensuados historiográficamente y los tiempos biográficos de los actores estudiados (Eduardo Ladislao Holmberg, Paul Groussac, José Manuel Estrada y Eduardo Wilde, centralmente). Se problematiza, además, la idea de que para dar miradas de conjunto sobre una época es necesario estudiar lo representativo o lo homogéneo, y se sugiere de qué formas el análisis biográfico puede dar cuenta de una trama cultural en una determinada coyuntura temporal.

Palabras clave: Biografía, Intelectuales, Vida cultural, Metodología.

\section{Biography and History of the Argentinean intellectuals. Balance and reflections of cultural life, 1860 y 1910}

\begin{abstract}
This article presents some reflections about a research experience on the Argentine cultural life in the second half of XIX century and the beginnings of the XX century, through comparative biographies. The article analyzes the possibilities of revising different items, such as: synchronicities between historical moments, historiographical consensus about periodizations and biographical times of the actors studied. Also, the paper questions the idea that states that in order to offer global images about a specific time period involves the study of representative features or homogeneic aspects. In this sense, the article suggests in which forms the use of biographies give an account of the cultural plot.
\end{abstract}

Keywords: Biography, Intellectuals, Cultural Life, Methodology.

\footnotetext{
* Agradezco a Claudia Darrigrandi y Lorena de la Paz Amaro por haberme animado a escribir este ensayo. El mismo fue redactado durante una estancia de investigación financiada por el Instituto Iberoamericano de Berlín.

** Argentina. Doctora en Historia por la Universidad de Buenos Aires. Investigadora del CONICET (Consejo Nacional de Investigaciones Científicas y Técnicas). Directora del Departamento de Estudios Históricos y Sociales de la Universidad Torcuato Di Tella. Directora de la Red de Estudios Biográficos de América Latina. Buenos Aires, Argentina. pbruno@conicet.gov.ar
} 
En un célebre pasaje, Marguerite Yourcenar (1994) apuntaba: «los historiadores nos proponen sistemas demasiado completos del pasado, series de causas y efectos harto exactas y claras como para que hayan sido alguna vez verdaderas; reordenan esa dócil materia muerta, y sé que aun a Plutarco se le escapará siempre Alejandro» (11). En un libro de reciente aparición, por su parte, Ivan Jablonka (2016) señala que los historiadores y los cientistas sociales suelen mostrar los resultados de sus investigaciones sin hacer explícito el revés de la trama. Como si las reglas del oficio generaran una falsa idea de «acabado» que no permiten ver cuáles fueron las dinámicas que llevaron a los resultados mostrados.

En este artículo, asumo el desafío que ambos pasajes sugieren, y comento algunas cuestiones ligadas con una investigación que hoy se conoce como libro, bajo el título Pioneros culturales de la Argentina. Biografías de una época, 1860-1910 (Bruno, 2011). Lejos del relato etnográfico o autobiográfico ligado a la investigación, me detengo en las reflexiones particulares que en este trabajo signaron las decisiones para pensar una época por medio de biografías. Sobre todo, centro la atención en tres cuestiones interconectadas: cómo se pueden repensar periodizaciones de procesos macro desde el abordaje biográfico; cómo es posible cuestionar lecturas que rigidizan definiciones de actores bajo rótulos abarcadores; $y$, por último, cómo se pueden encuadrar voces particulares en lo que convencionalmente se consideran climas corales.

Pensar una época por medio del rastreo de trayectorias vitales es una práctica extendida en diversas historiografías. Los modelos y las posibilidades metodológicas son varias (Dosse, 2007; Lee, 2009; Loriga, 2010). En las últimas décadas se produjo una revalorización del género biográfico en su sentido clásico -en tanto vidas de prohombres- inscripto en la renovación de la historia política (Levillain, 1998; Gueniffey, 2004), a través de estudios en los que desde una biografía no representativa se concreta un acercamiento a problemas epocales (Ginzburg, 1977) y otras en las que la figura biografiada sí condensa un momento paradigmático (Pocock, 1975; Rosanvallon, 1985).

Otras opciones estudian varios itinerarios sobre un telón de fondo compartido. Se utilizan varias trayectorias para estudiar tendencias diacrónicas encarnadas en personajes de distintas épocas y también sincronías entre biografías (Ozouf, 1995). En algunos casos se centra la atención en grandes personalidades históricas (Craveri, 2006), y en otros, en figuras en los márgenes (Zemon Davis, 1995). 
Una tercera posibilidad se encuentra en estudios de itinerarios singulares que dan cuenta dinámicamente de una época (Schorske, 1981; Charle, 1998; Mangoni, 1985; Lepenies, 1994; Darnton, 1991). Para iluminar la diversidad dentro de un cuadro de conjunto, el seguimiento de una variedad de figuras intelectuales se considera un medio eficaz en varias investigaciones. En esta alternativa se inscribió la propuesta de la mencionada investigación. Se estudió así un momento histórico reincidentemente visitado -la segunda mitad del siglo XIX y los inicios del XX-, pero que fue escasamente transitado desde el estudio de trayectorias intelectuales, y aún menos desde un seguimiento de vidas paralelas, parafraseando el célebre título de la obra de Plutarco.

De este modo, el objetivo de la indagación era dar cuenta de un momento de la vida cultural de la Argentina por medio de cuatro trayectorias individuales: Eduardo Wilde (Tupiza, 1844 - Bruselas, 1913), José Manuel Estrada (Buenos Aires, 1842 - Asunción, 1894), Paul Groussac (Toulouse, 1848 - Buenos Aires, 1929) y Eduardo Ladislao Holmberg (Buenos Aires, 1852-1937). Por medio del seguimiento de estas biografías de figuras intelectuales se reconstruyó la trama de una época y se trazó un mapa cultural y sus particularidades.

François Dosse (2007) se ha interrogado si es necesario revisar las vidas de los intelectuales, o, si con el estudio de sus obras sería suficiente para recuperar su valor cultural y su legado. En este sentido, el autor propone dar un espacio a la recepción de las obras de los intelectuales biografiados, pero, a la vez, reparar en las marcas y huellas biográficas que propiciaron que esas obras fueran escritas en momentos particulares e irrepetibles en las trayectorias de los mismos. Destaca, entonces, que la vida de un intelectual y su obra: «no pueden tratarse como si estuvieran separadas por tabiques estancos, ni tampoco reducirse a un solo nivel» (387). Atendiendo a este desafío, en la mencionada investigación, se estudiaron en paralelo los itinerarios vitales y las producciones intelectuales de las figuras seleccionadas.

La historiografía, la crítica literaria y otras disciplinas afines transitaron con asiduidad la esfera cultural de la etapa que lleva en Argentina el consensuado rótulo «del Ochenta al Centenario», es decir de 1880 a 1910. Es la etapa de la conformación del Estado, la construcción de la nación, la articulación del país con los mercados internacionales y la secularización, entre otros rasgos. 
El retrato y la caracterización de los intelectuales finiseculares asumieron una centralidad notable en los trabajos abocados a dichos horizontes de problemas ${ }^{1}$. Básicamente, en la bibliografía disponible las figuras y voces que recibieron mayor atención fueron, por un lado, las más clásicamente relacionadas con la llamada «generación del ochenta» -considerada una generación de conductores, no siempre adjetivada positivamente-, desde perspectivas que más que detenerse en las dinámicas culturales y en los intelectuales como hombres de ideas, los retrataron con elementos extra-culturales -principalmente la clase social-. Por otro, predominaron semblanzas vinculadas a un clima de ideas acompasado por la cultura científica y la cuestión nacional en sus distintas manifestaciones; en este sentido, se estudiaron los hombres ligados al auge del ensayo positivista, a las Ciencias Sociales o a los nacionalistas culturales del Centenario de 1910.

Por su parte, dentro de la bibliografía relevada sobre los intelectuales del cambio del siglo XIX al XX, en Argentina fueron cuatro los elementos centrales para pensar en las figuras, sus ideas y roles: la clase o pertenencia social, el Estado, la nación y el mercado. Estas cuatro referencias clásicas y fundamentales se combinan de diversa forma en los universos hermenéuticos de la producción académica (Altamirano, 2007).

Partiendo de este balance de la bibliografía disponible, fueron supuestos fuertes, derivados de los mencionados encuadres, los que encontré reiteradas veces y con los que medí, de alguna manera, las potencialidades y los límites para matizar o disentir con los consensos historiográficos vigentes a partir de las indagaciones biográficas.

En un momento inicial, asumí que la cronología consensuada, 18801910, contaba con una validez indiscutida. La convención cronológica proviene de la historia política que tiende, en general, a hacer un corte tajante entre el período comprendido entre 1852 y 1880 y el desplegado entre 1880 y el Centenario. De alguna forma, estas marcas temporales condicionan también las formas de pensar el resto de las esferas, la economía, la sociedad y sus dinámicas y el mundo cultural.

Se asume que la vida cultural posterior a la experiencia de Juan Manuel de Rosas (1829-1852) estuvo dominada por la omnipresencia de los «padres fundadores», los miembros de la generación del 37, que ha- 
bían sido exiliados, pero eran ahora figuras consolidadas. Se estudian sus perfiles y se describe un mundo político superpuesto al cultural; esto se da porque, justamente, los «padres fundadores», en tanto intelectuales y políticos, condujeron los destinos de la Argentina y dieron forma a sus instituciones. Las figuras centrales en este sentido fueron Juan Bautista Alberdi, Bartolomé Mitre y Domingo Faustino Sarmiento. Se los suele retratar como héroes o prohombres político-intelectuales. De este modo, sus figuras de gigantes parecen haber dejado secundarizadas todas las manifestaciones de la cultura que no estuvieran por ellos conducidas. Para los años posteriores a los de auge de estos personajes, y para la época abierta en 1880, se suele destacar un escaso brillo intelectual de los letrados que sucedieron a esa camada fundadora (Halperin Donghi «La historiografía» $\mathrm{y}$ «Un nuevo clima»).

Los hombres públicos del ochenta, por su parte, fueron generalmente pensados como pragmáticos mentores de proyectos y planes apoyados en las intenciones de orden, paz, administración y progreso, más allá del signo positivo o negativo con los que se cargaron estas caracterizaciones. Sobre todo, en las décadas de 1960 y 1970 cristalizaron fuertes prototipos de políticos-intelectuales vistos como los forjadores de una nueva era en la que el mundo de las ideas y la acción aparecían como dos caras indisociables de la misma moneda. En este sentido, consideraciones acerca de la adscripción de éstos a los intereses del Estado o de la clase social se refieren con frecuencia.

En el primer sentido, el uso de la palabra hace de los intelectuales «cómplices» o funcionarios del Estado formador de la nación. Varias caracterizaciones dan cuenta de esta interpretación: «intelectual-político», «hombre del régimen», «literato oficial», entre otras. Generalmente, como las expresiones mencionadas sugieren, se aceptó la caracterización del ochenta como un momento en el que la esfera de la cultura se confundió con el ámbito del poder, o se subordinó al mismo. De este modo, intelectualidad y élite política aparecen asociadas de manera casi automática y, por tanto, los intelectuales se consideran como engranajes del aparato estatal o de la clase política. Así, ciertos intelectuales-políticos con un alto grado de lucidez y claridad ideológica pasaron a ser los prototipos de clase de una generación hacedora del Estado y la nación.

Consideraciones sobre la adscripción de los intelectuales a los intereses del Estado conviven con frecuencia con caracterizaciones sobre las marcas de la pertenencia de clase. Éstas fueron pensadas hacia la déca- 
da de 1970, sobre todo, como determinantes en la definición de perfiles intelectuales (McGann, 1960; Viñas, 1964; Jitrik, 1968). En esta dirección, las tareas específicamente intelectuales de las figuras del ochenta no cuentan en estas interpretaciones con ningún tipo de gravitación en el marco general de las actividades de los hombres públicos de entonces, caracterizados como dandys que escriben o gentlemen escritores, entre otras rotulaciones.

Si en el primer sentido, el uso de la palabra convierte a los intelectuales en escribas y/o cómplices del Estado y formadores de la nación, en el segundo, el trabajo de ideas es entendido como una actividad secundaria, ociosa, o que se halla al mismo nivel que la concurrencia a un club social o al hipódromo. Paradójicamente, definiciones tan opuestas fueron atribuidas a los mismos nombres en algunas de las semblanzas más exitosas sobre los letrados de esta época; siendo, quizás, el caso paradigmático el de Miguel Cané 2 .

De la distancia de estas propuestas interpretativas, surgieron en mi indagación dos tipos de preguntas. Las primeras ligadas al corte cronológico «antes y después de 1880». Sobre el «antes»: ¿qué había más allá de los «padres fundadores» en la vida cultural del período post-rosista? Vale decir: ¿quiénes estuvieron frente a las cátedras, las revistas y las sociabilidades de entonces? La pregunta se encuadraba en la ponderación de una época: se trataba de un contexto en el que el Estado avanzaba y generaba espacios institucionales que requerían de nuevos hombres que ocuparan espacios en un ámbito cultural que no estaba organizado. El rosismo no legó instituciones asentadas, linajes intelectuales indiscutidos, espacios consolidados, sociabilidades, academias o institutos. Luego, partiendo de estas coordenadas: ¿quiénes ocuparon el nuevo mundo cultural que surgió hacia 1860 ?

Sobre el «después del ochenta»: ¿se dibujó hacia 1880 una homogeneidad ideológica, bajo el signo del orden y el progreso, que aplacó los debates de las décadas anteriores? ¿Se habían aplacado las querellas intelectuales? De no ser así: ¿cuáles fueron las prioridades para pensar la Argentina de las figuras intelectuales cuyas trayectorias se desplegaron entre 1860 y 1900, aproximadamente? 
Otra línea de preguntas se derivó de las coordenadas que se utilizan para pensar en los hombres de ideas del ochenta: su adhesión al Estado y su pertenencia de clase. Acerca del primer aspecto: ¿la idea de un Estado monolítico y aceitado y de intelectuales trabajando para esta maquinaria, se ajustaba a la configuración estatal del ochenta? Y, por otro lado: ¿cuáles fueron las miradas sobre el Estado y sobre las dinámicas de la política que los hombres de cultura legaron? ¿Era lo mismo ocupar puestos de funcionarios del Estado y adherir a las intenciones oficiales?

En otro sentido, sobre su pertenencia de clase: ¿el hecho de que los literatos formaran parte de la clase alta es condición suficiente para no considerarlos como hombres de ideas? Y, derivada de esta pregunta, otra: ¿todos los intelectuales provenían del mismo lugar social?

Al procurar dar respuesta a algunas de estas preguntas, y atendiendo a los tiempos de las biografías estudiadas y de la vida cultural, una primera decisión fue renunciar a la idea de un corte tan tajante entre las décadas 1860-1880 y 1880-1910 y apostar, en cambio, a estudiar el mediano plazo. En esta dirección, resultó fructífera la afirmación de Arcadio Díaz Quiñones (2006): «la biografía intelectual es una manera de romper con las cronologías políticas cristalizadas y con la sucesión de ismos característicos de la historiografía literaria, que a menudo terminan por deshistorizar su objeto o por limitar estrechamente el campo del individuo» (38). Los derroteros vitales habilitaban esta ampliación del corte cronológico. Este hecho conllevó a dos novedades. En primer lugar, a reconocer la convivencia de perfiles culturales variados que se desenvolvieron desde la década de 1860 en adelante.

Después de la batalla de Pavón (1861) se organizó una comunidad intelectual que sufría constantes modificaciones por la llegada de sabios y eruditos de otras latitudes, la convivencia de hombres de diversas edades, los reacomodamientos entre recién llegados y los ya establecidos. Los ejemplos de trayectorias disímiles en una esfera cultural que se estaba reconfigurando vertiginosamente son numerosos. Para resumir estas posibilidades, presento aquí sólo algunas referencias para pensar en la comunidad intelectual que se desenvolvió en estas décadas: con los hombres del interior y del exilio (por ejemplo: Eduardo Wilde, Héctor Varela, Lucio V. López y Miguel Cané) convivieron los porteños de familias arraigadas desde larga data en el país que no habían sufrido el destierro y el desarraigo en tiempos rosistas (se cuentan entre ellos José Manuel Estrada, Eduardo Ladislao Holmberg, Pedro Goyena, Lucio V. Mansilla 
y otros). Éstos, además, interactuaron con extranjeros que se sumaron armónicamente a los ámbitos culturales (piénsese, según el momento, en Paul Mortá, Amadeo Jacques, Alberto Larroque y Paul Groussac). Allí estuvieron también los padres fundadores y los hombres que ocuparon espacios centrales luego de las batallas de Caseros y Pavón (como Nicolás Avellaneda, Miguel Navarro Viola, Vicente Quesada, Estanislao del Campo, Hilario Ascasubi y otros). En este mapa cultural, ya de por sí variado, comenzaron a tener una presencia las figuras nacidas a lo largo de las presidencias desplegadas desde 1862 (como Martín García Mérou, Antonio Dellepiane, Ezequiel P. Paz y Ángel Estrada).

En segundo lugar, las preguntas me condujeron a renunciar a la «fotografía del ochenta». Es decir, a evaluar los roles intelectuales que se desplegaron con vigor en las décadas anteriores a la presidencia de Julio A. Roca (1880-1886); momento en el que distintas figuras actuaron como pioneros de la cultura. Así, por ejemplo, en lugar de pensar en el maduro José Manuel Estrada de la década de 1880 encarnando la voz de la resistencia ante las reformas laicas, se rastreó también su pasado en los ámbitos educativos: entre 1866 y 1868 dictó famosas lecciones de historia argentina en la Escuela Normal; durante la presidencia de Domingo Faustino Sarmiento (1868-1874) recibió un nombramiento como Jefe del Departamento General de Escuelas y fue Presidente del Congreso de Instrucción Pública. En 1874 fue Director de Escuelas Normales. En el Colegio Nacional de Buenos Aires ejerció como docente de las asignaturas de Instrucción Cívica e Historia Argentina y ocupó el cargo de Rector entre 1876 y 1883. Junto a ello, se desempeñó desde 1875 como catedrático de Derecho Constitucional y Administrativo en la Facultad de Derecho y Ciencias Sociales de la Universidad de Buenos Aires y el Congreso Pedagógico Nacional de 1882 lo tuvo como uno de sus vicepresidentes. Sólo su posición en el conflicto suscitado por las reformas laicas, que generó exoneraciones de sus cargos docentes en los primeros años de la década de 1880, lo apartó de la esfera educativa y lo empujó con decisión a la esfera política.

El mismo tipo de opción tomó forma al estudiar a quien es generalmente considerado como arquetípico hombre del roquismo, Eduardo Wilde. Si se deposita la mirada en su pasado se descubre a un hijo de padre exiliado en los tiempos del rosismo, que desde su ingreso a Buenos Aires, a comienzos de la década de 1860, participó en empresas intelectuales que fueron definiendo su perfil de hombres de letras. Antes del 
ochenta, Wilde fue cronista en periódicos, profesor en el Colegio Nacional y en la Facultad de Medicina, médico e higienista y demostró en cada una de sus actividades una vocación de intervención pública mediada por el trabajo intelectual. En estos años previos a 1880, no podría sostenerse que Wilde haya estado asociado de manera automática a la clase política ni mucho menos que formara parte de un círculo social distinguido. Aún así, su acercamiento a la pluma y su vocación de intervención se manifestó con constancia ${ }^{3}$.

Considerar estos rasgos de más largo plazo, permite entonces, matizar la caracterización del gentleman escritor sólo como manifestación de un tipo social o miembro de un régimen político, e invita a la reflexión acerca de cuáles fueron los rasgos de los hombres de ideas en la sociedad argentina de la segunda mitad del siglo XIX. Sugiere con fuerza, además, otro de los puntos en los que me interesa detenerme en estas páginas, el del uso de las biografías y la comparación.

En las décadas comprendidas entre la revolución de mayo de 1810 y la consolidación del rosismo pueden reconocerse y caracterizarse espacios de sociabilidad de manera relativamente precisa e incluso mencionar con precisión a las figuras del círculo letrado porteño -piénsese en la Sociedad Patriótica y los hombres de la revolución, en la Sociedad Literaria de Buenos Aires y el grupo rivadaviano y en el Salón Literario y la generación del 37-. Sin embargo, hacia la década del sesenta del mil ochocientos, identificar a un solo grupo o describir un único espacio de sociabilidad intelectual preponderante no es posible (Bruno, 2014). De este modo, si se confrontan los años post-186o con los decenios anteriores, salta a la vista que la novedad central de esta etapa es la apertura de una multiplicidad de zonas culturales en el ámbito porteño. Estas zonas, como ya se señaló, estuvieron habitadas por perfiles variados. Los intelectuales que desplegaron sus tareas a partir de la década de 1860 dieron sus pasos iniciales en un contexto en el que los antaño hombres de la generación del 37, ahora padres fundadores, ocupaban la escena con determinación. Ese grupo intelectual de antecesores se definió por elementos comunes: su formación en tiempos rivadavianos, la sociabilidad y la escritura de textos programáticos, el exilio, el antirrosismo ferviente y la voluntad de proyectar la nación.

3 La obra reunida de Eduardo Wilde puede consultarse en XIX tomos publicados por diferentes casas editoriales entre 1917 y 1939. 
El ocaso de las trayectorias que estudié se produjo entre la última década del siglo XIX y comienzos del XX. En esa etapa se profundizó la modernización del mundo cultural argentino y cambiaron los rasgos de su comunidad intelectual. Los cultores de las ciencias sociales y los adalides del ensayo positivista, por un lado, y las nuevas figuras profesionales y especializadas, por otro y apenas más tarde, se emplazaron en lugares más pautados que los preexistentes. Un nuevo traje de intelectual trajo consigo nuevas formas de usar la palabra. Se definieron vocabularios y aparatos críticos compartidos para decir la sociedad y se estabilizaron pautas de circulación y aceptación de saberes.

La historiografía, entonces, por una parte, se detuvo a estudiar el mundo post-Caseros centrándose en el indiscutido peso de los «padres fundadores». Por otra, se centró la mirada en el cambio de siglo, momento en el que se profundizó la modernización del mundo cultural argentino y se delineó con más claridad la profesionalización de las tareas intelectuales -piénsese en los institutos, las academias, las facultades, los grupos de referencias, las revistas, las redes intelectuales-, ahora ejercidas por cultores de saberes doctos y profesionales. En el marco de estas lecturas, los ritmos culturales de las décadas que van desde 1860 hasta 1900 no han acaparado atención sistemática, tal vez porque se consideraron como un período de opacamiento de la vida intelectual, siguiendo la caracterización propuesta por Halperin Donghi («La historiografía: treinta años en busca de un rumbo»).

Entonces, surge la siguiente inquietud: si las figuras preponderantes de la generación del 37 marcaron los ritmos del post-rosismo, y las de los ensayistas positivistas y los hombres del Centenario definieron el cambio de siglo: ¿qué elementos caracterizaron a las figuras intelectuales que tuvieron protagonismo entre 1860 y 1900, aproximadamente? Definir una constelación intelectual que se despliega en un terreno fecundo y de apertura cultural, y que además se encuentra entre dos momentos de rasgos estudiados de manera sistemática por la historiografía argentina, no es una tarea sencilla.

Frente a este panorama, la posibilidad de entender a los intelectuales estudiados como «figuras en transición» fue tentadora. Podría incluso haber sido efectiva. Por ejemplo, se podría haber aseverado que Groussac estuvo a mitad de camino entre un historiador romántico y un historiador profesional; que Estrada fue el eslabón intermedio entre los letrados católicos como el Deán Gregorio Funes o Félix Frías y los intelectuales 
católicos del siglo XX, como Gustavo Franceschi; que Holmberg fue una figura estrafalaria entre el explorador amateur y el científico profesional. Se podría haber aceptado que estas figuras en transición se desplegaban en una especie de «limbo cultural». Como si entre la generación del 37 y el cambio de siglo los hombres de letras sólo hubiesen podido definirse culturalmente de manera imprecisa. Sin embargo, en lugar de asumir esta mirada, se optó por historizar este momento particular de la Argentina por medio del seguimiento de biografías. En este punto, la necesidad de ampliar la cronología y salir del recorte «ochenta al Centenario» confluyó armónicamente con mi interés particular por la biografía como forma de aproximación al pasado.

Para iluminar la diversidad dentro de un cuadro época, estudié un momento histórico reincidentemente visitado -la segunda mitad del siglo XIX y los inicios del XX-, desde una perspectiva biográfica y atenta a las improntas personales que se desplegaron en un cuadro compartido. A su vez, inscribí las semblanzas en universos más amplios por medio del juego de comparaciones y confrontaciones con otras figuras ya sea contemporáneas o antecesoras y posteriores.

Como es sabido, la búsqueda de personajes representativos que ilustran las grandes ideas de la época, es decir, de voces dominantes y paradigmáticas, ha atravesado y caracterizado a las producciones que se ocupan de la vida intelectual del período. En cambio, las biografías comparadas que realicé se basaron en un proyecto de investigación que no partió de la búsqueda de figuras y voces que ilustraran una sola vertiente de ideas (por ejemplo: «los conservadores», «los nacionalistas», etc.), ni que se congregaran en un mismo ámbito de sociabilidad o fueran los promotores de una única empresa cultural («los hombres del Ateneo cual» 0 «los escritores de la revista tal»), ni que estuvieran ligadas indiscutiblemente por su pertenencia a una facción política (por ejemplo: «los roquistas» o los «mitristas»), o a una única franja de la cultura («los científicos», «los artistas plásticos»), sino más bien todo lo contrario. La investigación surgió de la intención de no evaluar bloques compactos de intelectuales adscriptos a una vertiente, una ideología o una empresa, sino más bien de recuperar posibilidades diferentes en el marco de una trama cultural compartida. En este punto, se fue definiendo con fuerza la figura de la «constelación intelectual» como una posibilidad para pensar puntos únicos, pero conectados. Estudié, de esta forma, trayectorias que dan cuenta del contexto, pero no lo agotan. 
Se apostó, de este modo, a presentar biografías de intelectuales -y no solamente biografías intelectuales- y a compararlas en un escenario cultural que se reveló múltiple y efervescente. Con este objetivo, se combinaron rasgos y circunstancias biográficas con tramas sociales y culturales. Se tuvieron en cuenta las formaciones educativas, sociabilidades, inserciones institucionales, participaciones en publicaciones periódicas y distintas formas de intervención pública. La información fue obtenida de archivos privados, periódicos, artículos, ensayos interpretativos, testimonios de contemporáneos y de las generaciones posteriores.

Para elegir las figuras busqué que se ubicaran en franjas culturales diferentes y, además, prioricé el relevamiento de voces que se distinguieran de aquellas cuya producción intelectual estuvo fuertemente encorsetada por conceptos, teorías o modelos explicativos rígidos que pretendieron fundar lecturas científicas (o legitimadas por el discurso científico) o que produjeran conocimiento sobre la sociedad de manera sostenida y sistemática. En este sentido, reparé en voces no siempre convencionales que muchas veces legaron representaciones sobre la sociedad de su tiempo por medio de pinceladas descriptivas, cuadros de costumbres y otros trazos. Rastreé, entonces, voces individuales que expresaron los clásicos tópicos de su tiempo, pero también otros menos transitados o más sesgados. Las particularidades de los argumentos puestos de relieve permitieron ver cómo la expresión de problemas de época fue tratada de manera diferencial y encontró respuestas múltiples en el marco de una Argentina caracterizada por el cambio como una constante.

Sobre este aspecto, quisiera hacer las últimas consideraciones de este ensayo. Las preguntas que guiaron el rastreo de estas voces tenían que ver con una pregunta surgida también de consensos historiográficos: si en las agendas de los letrados del exilio y la generación del 37 la preocupación central fue cómo crear una nación para el «desierto» argentino (parafraseando el título de un libro de Halperin Donghi), y en la agenda de los intelectuales del cambio de siglo las preocupaciones estaban focalizadas en los efectos de las multitudes cosmopolitas: ¿cómo pudo pensarse en la configuración social de un país desplegado entre estas estaciones? ¿Se trataba de un momento idílico en el que el progreso material todo lo resolvía? ¿Cuáles eran las preocupaciones de los hombres de cultura de esas décadas? ¿Cuál fue la agenda de problemas de aquellos años? ¿Hubo una o varias? ¿Se postulaban diferencias debajo del clima coral? 
La revisión de estos supuestos se vio beneficiada por varios frentes. Por un lado, en las últimas tres décadas se produjo en Argentina una revisión sistemática y profunda sobre la historia de las élites del cambio de siglo. Como resultado, connotaciones que habían ritmado las interpretaciones respecto del pasaje del siglo XIX al XX, como «clase terrateniente», «generación del 80», «oligarquía», «aristocracia», «clase dominante» o «roquismo» fueron revisadas y puestas en jaque a la hora de explicar dinámicas políticas, sociales y económicas (Hora, 2002; Losada, 2008). De este modo, grupos de actores históricos que anteriormente eran vistos como bloques compactos y uniformes ideológica y socialmente, pasaron a ser evaluados en sus fracturas y tensiones. Se comenzaron a estudiar conjuntos menos homogéneos de políticos, actores sociales y económicos que no fueron protagonistas únicos ni excluyentes en el marco de un país en plena transformación. Más cerca del terreno de la vida intelectual y cultural, se comenzaron a utilizar rótulos como «liberales reformistas» (Zimmemann, 1995), «patriotas, cosmopolitas y nacionalistas» (Bertoni, 2001), «primeros modernos» (Malosetti Costa, 2001), representantes de la «cultura científica» (Terán, 200o) e «intelectuales y expertos» (Plotkin y Neirbug, 2004). Todas ellas, denominaciones que organizan seguimientos de elencos más pequeños de hombres públicos. Con todo, puede señalarse que, de alguna forma, estos estudios redujeron la escala de observación en la historia política y cultural del período.

Esta tendencia comenzó a revertir, al menos en parte, la línea interpretativa comentada que reinaba, que sostenía que la cultura del período no contaba con ritmos propios hasta 1910, momento en el que se desplegó el proceso de profesionalización de las disciplinas intelectuales. Este supuesto, al asumir que la cultura había estado subordinada a los tiempos políticos -o a sus necesidades-, había dejado invisibilizados los márgenes de autonomía de ciertas dinámicas culturales y de los actores que las ponían en práctica. A su vez, se tendió a relevar un clima colectivo de ideas que se habría cristalizado en el año 1880, con grillas dadas por el positivismo o la cultura científica, que daba cuenta de problemas coreados: las lecturas sobre la configuración de una sociedad plural ritmada por la inmigración y el fenómeno «multitudinario», la preocupación por la nacionalización y la construcción de la identidad nacional, la cuestión obrera y/o social, los desafíos de la apertura política y los peligros de los efectos no deseados de la modernización. Mi interés fue mostrar las voces de intelectuales que, atravesados por los climas de época, no responden tan claramente a 
las figuras de positivistas, cientistas sociales, ensayistas, sino más bien de aquellos que llegan a sus reflexiones sobre la sociedad por lugares menos pautados y por una variedad de registros (crónicas, textos miscelánicos, ficciones, columnas de opinión en periódicos, textos eruditos publicados en revistas, entre otros).

Con la intención de dar cuenta de estas posibilidades, no se relevaron las voces por medio de un inventario de bibliotecas o de una genealogía de influencias y autoridades. Se buscó, en cambio, dar cuenta de las representaciones de la Argentina que estos intelectuales legaron atendiendo a las ideas y marcos de referencias utilizados para leer a la sociedad de su tiempo y construir sus propias voces. Por un lado, se estudiaron las miradas sociales que Wilde, Groussac, Holmberg y Estrada legaron $\mathrm{y}$, complementariamente, los diálogos entre los climas generales y las formas singulares de apropiación de los mismos. La convicción de que los intereses y vocabularios comunes de una época conforman lo que Mario Isnenghi (1979) denominó «esferas de pensamiento privilegiadas» guió el trabajo para visualizar el uso de un repertorio compartido e ideas rectoras (32). En este sentido, las voces de los intelectuales estudiados mostraron la coexistencia de pluralidad de discursos sociales y revelaron una imagen diferente a la del clima de ideas homogéneo. De este modo, se demostró, por ejemplo, que el uso de un repertorio de expresiones con acentos spencerianos y darwinianos a la hora de buscar respuestas a las realidades político-sociales del cambio de siglo no tuvo respuestas unívocas; y que los diagnósticos fatalistas sobre los «desencuentros de la modernidad»-la expresión es de Julio Ramos- o ante los efectos no deseados del progreso y la modernización no necesariamente derivaron en bloque en los tonos apesadumbrados del ensayo cientificista -que tendía a pensar a los países latinoamericanos como espacios condenados a un futuro fatídico-, o en programas de nacionalismo cultural -que consideraban que los programas de formación de una identidad nacional solucionarían diferencias generadas en un país de inmigración masiva-.

De este modo, relevar las particularidades de los argumentos que a primera vista se asumen como unitarios permite ver cómo la expresión de problemas de época y realidades sociales fue tratada de manera diferencial y encontró respuestas variadas para una Argentina caracterizada por el cambio como una constante. En suma, se logró dar cuenta una polifonía coral más que una voz monocroma. 
Así, las elecciones metodológicas condujeron a transitar la cultura tras las huellas de personajes constitutivos de la misma y dar cuenta de algunos rasgos particulares en relación con las trayectorias elegidas. Con una apuesta por la biografía, más que dar cuenta de itinerarios representativos para ilustrar tópicos, problemas o climas de la época identificables o reconocibles a priori, y con el objetivo de mostrar la convivencia y variedad de perfiles y voces de la época estudiada, seleccioné figuras que permitieran transitar diferentes franjas culturales. En este sentido, siguiendo a Carlo Ginzburg (1984) - y a los microhistoriadores en general-, se priorizó la representatividad histórica de los sujetos estudiados, que tiende a mostrar posibilidades dentro de un contexto, en lugar de la representatividad estadística, en la convicción de que la carencia de representatividad estadística no significa ausencia de historicidad ${ }^{4}$. De este modo, las trayectorias y voces estudiadas no son modelos generalizables, sino muestras de posibilidades en un escenario poroso y constitutivamente diverso. A partir de estas decisiones, los resultados no se detuvieron en dar cuenta de excentricidades, rarezas o fenómenos en los márgenes; en cambio, se reconstruyó una época, un cuadro de conjunto, sin deslucir el vigor de las singularidades y las particularidades.

\section{Referencias bibliográficas}

Altamirano, Carlos. Intelectuales. Notas de investigación. Buenos Aires, Grupo Editorial Norma, 2007.

Bertoni, Lilia Ana. Patriotas, cosmopolitas y nacionalistas. La construcción de la nacionalidad argentina a fines del siglo XIX. Buenos Aires, Fondo de Cultura Económica, 2001.

Bruno, Paula. Pioneros culturales de la Argentina. Biografías de una época, 1860-1910. Buenos Aires, Siglo XXI, 2011.

. «Vida intelectual de la Argentina de fines del siglo XIX y comienzos del XX. Un balance historiográfico». PolHis. Revista Bibliográfica del Programa Interuniversitario de Historia Política, no. 9, 2012, pp. 69-91.

Charle, Chritophe. Paris Fin-de-siècle. Culture et politique. Paris, Éditions du Seuil, 1998.

Sobre la «representatividad estadística» y la «representatividad histórica», véase Ginzburg, 2006. 
Craveri, Benedetta. Amantes y reinas. El poder de las mujeres. México, Fondo de Cultura Económica, 2006.

Darnton, Robert. Edition et sedition. L'univers de la litterature clandestine au XVIIIe siecle. Paris, Gallimard, 1991.

Díaz Quiñones, Arcadio. Sobre los principios. Los intelectuales caribeños y la tradición. Bernal, Editorial de la Universidad Nacional de Quilmes, 2006.

Dosse, François. La apuesta biográfica: escribir una vida. Trad. Joseph Aguado y Concha Miñana. Valencia, Universidad de Valencia, 2007.

Ginzburg, Carlo. El queso y los gusanos. El cosmos según un molinero del siglo XVI. Barcelona, Muchnik, 1997 (1976).

. «Prove e posibilita (Postfazione a Natalie Zemno Davis, Il ritorno di Martin Guerre. Un caso di doppia identità nella Francia del Cinquecento, 1984)». Il filo e le tracce. Vero falso finto. Milán, Feltrinelli, 2006, pp. 295-315.

Gueniffey, Patrice. «La biografía y la renovación de la historia política». La fuerza y el derecho. Estado, poder y legitimidad durante el siglo XVII. México, Centro de Estudios Históricos/El Colegio de México, 2004, pp. 81-97.

Halperin Donghi, Tulio. «La historiografía: treinta años en busca de un rumbo». La Argentina: del Ochenta al Centenario. Comp. Gustavo Ferrari y Ezequiel Gallo. Buenos Aires, Sudamericana, 1980a, pp. 829-840.

«Un nuevo clima de ideas». La Argentina: del Ochenta al Centenario.

Comp. Gustavo Ferrari y Ezequiel Gallo. Buenos Aires, Sudamericana, 1980b, pp. 13-24.

Hora, Roy. Los terratenientes de la pampa argentina. Una historia social y política, 1860-1945. Buenos Aires, Siglo XXI, 2002.

Isnenghi, Mario. Intellettuali militanti e intellettuali funzionari. Appunti sulla cultura fascista. Turín, Einaudi, 1979.

Jablonka, Ivan. La historia es una literatura contemporánea. Manifiesto por las ciencias sociales. Buenos Aires, Fondo de Cultura Económica, 2016.

Jitrik, Noé. El 8 o y su mundo, presentación de una época. Buenos Aires, Editorial Jorge Álvarez, 1968. 
Lee, Hermione. Biography. A very short introduction. Oxford, Oxford University Press, 2009.

Lepenies, Wolf. Las tres culturas. La sociología entre la literatura y la ciencia. México, Fondo de Cultura Económica, 1994.

Levillain, Philippe. «Les protagonistes: de la biographie». Pour une historire politique. Dir. René Rémond. Paris, Éditions du Seuil, 1988, pp. 121-159.

Loriga, Sabina. Le petit x: de la biographie à l'histoire. Paris, Seuil, 2010.

Losada, Leandro. La alta sociedad en la Buenos Aires de la Belle Époque. Sociabilidad, estilos de vida e identidades. Buenos Aires, Siglo XXI/Iberoamericana, 2008.

Malosetti Costa, Laura. Los primeros modernos. Arte y sociedad en Buenos Aires a fines del siglo XIX. Buenos Aires, Fondo de Cultura Económica, 2001.

Mangoni, Luisa. Una crisi fine secolo. La cultura italiana e la Francia fra Otto e Novecento. Turín, Eunaudi, 1985.

McGann, Thomas. Argentina, Estados Unidos y el sistema interamericano, 1880-1914. Buenos Aires, Eudeba, 1960.

Neiburg, Federico y Plotkin, Mariano (comp.). Intelectuales y expertos. La constitución del conocimiento social en Argentina. Buenos Aires, Paidós, 2004.

Ozouf, Mona. Les mots des femmes. Essai sur la singularité française. Paris, Fayard, 1995.

Pocock, John Greville Agard. The Machiavelian Moment. Florentine Political Thought and the Atlantic Republican Tradition. Princeton, Princeton University Press, 1975.

Rosanvallon, Pierre. Le moment Guizot. Paris, Gallimard, 1985.

Schorske, Carle E. Fin-de-siècle Vienna. Politics and culture. Nueva York, Vintage Books, 1981.

Terán, Oscar. Vida intelectual en el Buenos Aires fin-de-siglo (1880-1910). Derivas de la «cultura científica». Buenos Aires, Fondo de Cultura Económica, 2000.

Viñas, David. Literatura argentina y realidad politica. Apogeo de la oligarquía. Buenos Aires, Editorial Jorge Álvarez, 1964.

Yourcenar, Marguerite. Memorias de Adriano. Barcelona, Salvat, 1994. 
Zemon Davis, Natalie. Mujeres en los márgenes. Tres vidas del siglo XVII. Madrid, Ediciones Cátedra/Universitat de València, 1995.

Zimmermann, Eduardo. Los liberales reformistas. La cuestión social en la Argentina, 189o/1916. Buenos Aires, Sudamericana/UdeSA, 1995. 